\title{
LE MÉTIER DE MANAGER EN TRANSFORMATION : UNE DÉMARCHE PROSPECTIVE
}

Marine de Ridder, Laurent Taskin, Michel Ajzen, Marie Antoine et Chloé Jacquemin

Management Prospective Ed. | « Management \& Avenir »

2019/3 N 109 | pages 37 à 60

ISSN 1768-5958

Article disponible en ligne à l'adresse :

https://www.cairn.info/revue-management-et-avenir-2019-3-page-37.htm

Distribution électronique Cairn.info pour Management Prospective Ed..

(C) Management Prospective Ed.. Tous droits réservés pour tous pays.

La reproduction ou représentation de cet article, notamment par photocopie, n'est autorisée que dans les limites des conditions générales d'utilisation du site ou, le cas échéant, des conditions générales de la licence souscrite par votre établissement. Toute autre reproduction ou représentation, en tout ou partie, sous quelque forme et de quelque manière que ce soit, est interdite sauf accord préalable et écrit de l'éditeur, en dehors des cas prévus par la législation en vigueur en France. Il est précisé que son stockage dans une base de données est également interdit. 


\title{
Le métier de manager en transformation : une démarche prospective ${ }^{1}$
}

\author{
Marine DE RIDDER ${ }^{2}$ \\ Laurent TASKIN ${ }^{3}$ \\ Michel AJZEN ${ }^{4}$ \\ Marie ANTOINE 5 \\ Chloé JACQUEMIN 6
}

\section{Résumé}

Proposant d'actualiser la caractérisation traditionnelle du métier de manager en rôles et activités, cet article décrit le métier de manager aujourd'hui au travers d'une grille de compétences et emprunte une démarche prospective permettant de proposer un scénario d'évolution du métier de manager pour le futur. Nous montrons que ce métier sera soumis à trois champs de tensions particuliers: en matière de responsabilité de la décision, en particulier, de combinaison

1. Cette recherche a été financée par la Chaire laboRH en Management humain et transformations du travail de l'UCLouvain.

2. Marine DE RIDDER: Chercheuse, UCLouvain, Louvain Research Institute in Management and Organizations, CIRTES, Chaire laboRH marine.deridder@uclouvain.be

3. Laurent TASKIN : Professeur en Management humain et des organisations, UCLouvain, Louvain Research Institute in Management and Organizations, CIRTES, Chaire laboRH - laurent.taskin@uclouvain.be

4. Michel AJZEN : Chercheur, UCLouvain, Louvain Research Institute in Management and Organizations, CIRTES - michel.ajzen@uclouvain.be

5. Marie ANTOINE: Docteure en sciences économiques et de gestion (UCLouvain), chercheuse au LENTIC, HEC Liège - Ecole de gestion de l'Université de Liège, et professeure invitée à l'UCLouvain - m.antoine@uclouvain.be

6. Chloé JACQUEMIN: Chercheuse, UCLouvain, Louvain Research Institute in Management and Organizations, CIRTES, Chaire laboRH chloe.jacquemin@uclouvain.be 
d'une expertise gestionnaire et technique, de gestion des individualités et de fédération d'un collectif.

\begin{abstract}
In this paper, we first offer an update of the traditional characterization of the profession of manager (by activities or roles). We describe the actual profession of manager through a competencies grid. We adopt a provisional prospective approach enabling the establishment of an envisaged scenario of the future profession of manager. We highlight that this profession will certainly be characterized by three specific tensions: the accountability of decisions; the combination between technical and managerial expertise; the need of individual management and the need of federating a collective.
\end{abstract}

\title{
Introduction
}

À l'horizon 2030, 40 à $65 \%$ des emplois actuels n'existeront plus et $85 \%$ des métiers qui seront alors exercés n'existaient pas encore en 2017. Ce sont, en tous cas, les affirmations d'études prospectives menées par diverses organisations (Adecco, 2017 ; Institute for the Future \& Dell Technologies, 2017; McKinsey Global Institute, 2017; parmi d'autres), et largement commentées et relayées dans les médias. Si la digitalisation et la robotisation d'activités qui étaient confiées traditionnellement à des humains sont les facteurs les plus cités pour justifier cette mutation radicale, d'autres transformations sont aujourd'hui à l'œuvre et semblent affecter le travail. Parmi celles-ci, citons les enjeux de diversités (âge, sexe, culture et culte) et d'égalité qui en découlent, la transformation de la posture des individus par rapport au travail (effet générationnel, diminution du temps de travail...) ou de démocratisation du gouvernement des entreprises (holacratie, entreprise libérée ou agile et autres formes d'autogestion). Autant d'enjeux sociétaux et organisationnels qui affectent l'organisation du travail et la gestion de l'entreprise et, donc, le métier de manager.

Traditionnellement caractérisé au regard d'activités et de rôles, en référence aux travaux de Taylor (1911), Fayol (1916), Weber (1947) puis de Mintzberg (1975), l'étude du métier de manager se résume souvent à l'identification d'activités types que sont : (i) la gestion et le pilotage des activités et de la performance, (ii) la gestion des équipes et des personnes, (iii) la contribution à la stratégie par la planification, et (iv) la gestion du changement (Payre, 2010). Il est étonnant de constater une telle stabilité dans la caractérisation du métier de manager dans un contexte que d'aucuns décrivent comme étant en « profonde mutation » et insatisfaisant si l'on se réfère aux derniers chiffres de l'enquête « Parlons Travail » (CFDT, 2017). Le métier de manager serait-il immunisé de ces transformations sociétales et du travail ? À y regarder de plus près, il semble 


\section{Le métier de manager en transformation : une démarche prospective}

que le management soit tantôt appelé à disparaître en tant que métier — ainsi, pour certains tenants de l'entreprise libérée, la fonction est partagée par les travailleurs - soit, au contraire, à se réinventer au regard des nouveaux enjeux auxquels les organisations font face : déshumanisation, diversité, travailleurs plus qualifiés ou encore individualisation (Taskin \& Dietrich, 2016). Comment, toutefois, rendre compte de la transformation à venir du métier de manager sans se munir d'une cartographie contemporaine?

Cet article part du constat du manque d'adéquation entre les caractérisations existantes du métier de manager et le travail réel de l'encadrement tel qu'il se pratique dans les organisations, d'une part, ainsi que de la nécessité de développer une analyse prospective, d'autre part (Payre \& Scouarnec, 2015). Pour ce faire, dans un premier temps, nous caractérisons le métier de manager aujourd'hui, en prenant en considération les travaux traditionnels mais aussi les plus récents sur le sujet. Afin de compléter cette caractérisation, cinq entretiens semi-directifs approfondis ont été réalisés auprès de managers expérimentés que nous avons interrogés sur l'évolution de leur métier ces vingt dernières années. Intégrant une perspective dynamique aux typologies existantes, une grille de compétences est arrêtée et caractérise le métier de manager aujourd'hui. Dans un second temps, trois focus groups ont été menés avec des experts, DRH, PDG ; des managers ; et des salariés, afin d'appréhender les transformations perçues et souhaitées du métier de manager, du point de vue de ces principales parties prenantes.

Dans une première section, la littérature portant sur le métier de manager est présentée. Afin de poursuivre cette actualisation, les résultats des cinq entretiens approfondis sont présentés et rendent compte des transformations vécues par ces managers expérimentés. Finalement, une grille de compétences caractérisant le métier de manager est proposée. Dans une seconde section, la démarche prospective est présentée et les résultats des focus groups sont exposés. Cette section se focalise sur quatre dimensions qui semblent caractériser le métier de manager à l'horizon 2030. Finalement, les tensions inhérentes à l'exercice du métier de manager sont discutées d'un point de vue méthodologique et des implications pour la pratique et la formation du management.

\section{Caractériser le métier de manager}

Habituellement, le manager est défini comme celui «ayant la responsabilité d'une organisation ou d'une des unités de cette dernière » (Mintzberg, 1984, p. 32). Cette notion est à distinguer de la notion de leader. Le leader est plutôt associé à l'idée de changement et d'innovation (Knights \& Willmott, 2012). Il crée du changement organisationnel afin de susciter des nouveaux modèles d'action et de nouveaux systèmes de valeurs (Barker, 1997). Le manager, lui, anticipe le changement et y réagit mais ne le crée pas (Knights \& Willmott, 2012). 


\subsection{Activités et rôles}

Traditionnellement, le métier de manager est appréhendé selon deux approches : par les activités et par les rôles. Dans la première perspective, le métier de manager est caractérisé par un processus composé de cinq activités : planifier, organiser, commander, coordonner et contrôler (Fayol, 1916). Cette manière de décrire le management, qui date du début du siècle dernier, est encore utilisée aujourd'hui comme cadre de référence (Korica, Nicolini \& Johnson, 2015), même si certaines variantes ont vu le jour. Ainsi, Robbins et ses collègues (2011) préfèrent les termes suivants : planifier, organiser, diriger, et contrôler. Dans ce processus, la planification reprend la définition des objectifs et la mise en place des stratégies nécessaires à la réalisation de ceux-ci. L'activité d'organisation consiste à définir les tâches à réaliser et les personnes qui en seront responsables. Le commandement fait référence aux activités de direction et de motivation des collaborateurs mais aussi à la coordination des différentes tâches à effectuer ou encore à la gestion des conflits interpersonnels. Enfin, le contrôle consiste à s'assurer de l'obtention des résultats attendus. Ces quatre activités sont menées de front, ne peuvent pas être dissociées les unes des autres ou séparées dans le temps. Finalement, elles s'articulent autour de l'atteinte des objectifs fixés par l'organisation.

La seconde approche se réfère aux rôles et est généralement associée à Henry Mintzberg (1984). Celui-ci s'inspire des activités de Fayol (1916) et propose dix rôles pour caractériser le métier de manager. Mintzberg (1984, p. 65) précise que : «la personnalité individuelle peut avoir une influence sur la façon dont le rôle est tenu, mais ne peut pas empêcher que le rôle soit tenu. Ainsi, les managers et d'autres personnes sont les interprètes de rôles prédéterminés mais individuellement, ils les incarnent de diverses façons ». Mintzberg (1984) affirme également que les managers ont en commun d'être investis d'une autorité formelle leur conférant un statut particulier au sein de l'organisation. De ce statut découlent trois catégories de rôles hiérarchisées qui sont reliées entre elles. Premièrement, le statut conféré par l'autorité formelle conduit les managers à trois formes de rôles interpersonnels : les rôles de symbole, de leader et d'agent de liaison. Ensuite, les relations interpersonnelles induisent l'accès à l'information et les rôles y étant liés : les rôles d'observateur actif, de diffuseur et de porte-parole. Enfin, l'accès à l'information pose les bases du processus de prise de décision et permet au manager d'assumer quatre rôles décisionnels : les rôles d'entrepreneur, de régulateur, de répartiteur de ressources et de négociateur. Comme dans le processus de management de Fayol (1916), les rôles du manager sont concomitants : un manager est constamment impliqué dans des rôles décisionnels, interpersonnels, et liés à l'information. De plus, Mintzberg (1984) précise que les rôles forment une Gestalt, c'est-àdire un ensemble dont les parties sont indissociables les unes des autres. Les activités liées au management de Fayol (1916) se retrouvent dans les différents rôles du manager : la planification se retrouve dans le rôle de répartiteur des 


\section{Le métier de manager en transformation : une démarche prospective}

ressources, la direction se prolonge dans le rôle de leader, le contrôle est lié au rôle de régulateur, etc.

Plus récemment, d'autres auteurs se sont intéressés au métier de manager et à sa caractérisation. Ainsi, Hales (1986) se focalise sur l'étude des tâches, des responsabilités et des fonctions des managers. Il propose de distinguer, d'une part, les activités et les comportements des managers (ce que les managers font) et, d'autre part, les tâches, les responsabilités et les fonctions des managers (ce que les managers sont chargés d'accomplir). Cette approche plus récente permet de pointer les responsabilités, les fonctions et les activités communes à tous les managers. En se basant sur un siècle de recherches sur les managers, Hales (2001) propose des activités communes à tous les managers telles qu'agir comme le représentant ou le point de contact d'une unité de travail, diffuser l'information, s'engager dans des opportunités de réseautage ou encore négocier, planifier le travail, allouer des ressources entre différentes activités de travail, diriger et surveiller le travail des subordonnés, etc. Il caractérise également ce métier par la fragmentation des activités, un grand nombre d'interactions verbales souvent en présentiel et des tensions ou de la pression résultant de la volonté de répondre à différentes demandes concurrentes.

La transformation des organisations bureaucratiques reposant sur une hiérarchie verticale, une grande formalisation, et un contrôle structurel vers des nouvelles organisations post-bureaucratiques, ou postmodernes selon Lacan (2016), caractérisées entre autres par la responsabilisation des travailleurs, la confiance, le dialogue et des pratiques de flexibilité diverses entraînent la transformation des activités et des rôles traditionnels des managers (Grey \& Garsten, 2001; Taskin \& Dietrich, 2016). Ces changements défient le caractère intemporel et stable des rôles proposés par Mintzberg (1984). En effet, les rôles traditionnels des managers basés sur le « commander et contrôler » sont appelés à évoluer (e.g. Holtz, 2017 ; Dejoux \& Léon, 2018). Dans la littérature francophone, plusieurs auteurs se sont intéressés à l'évolution du métier de manager dans les organisations (e.g. Payre \& Scouarnec, 2015) comme Dumazert $(2011,2017)$ qui montre les futurs possibles existants entre le métier de manager et les chefs de guilde dans les milieux virtuels.

Aujourd'hui, il semblerait que les managers soient amenés à penser au-delà des silos organisationnels et à jouer le rôle d'intégrateur entre différentes équipes et de facilitateur dans la responsabilisation des employés (Barley \& Kunda, 2001 ; Kanter, 1989). Ils sont également invités à promouvoir l'apprentissage organisationnel, à inspirer, à inciter au changement (Hales, 2001, 2002) et à être porteurs de sens (Lacan, 2016). Les composantes d'inspiration et de changement rejoignent la transformation des discours managériaux basés sur la distinction entre manager et leader en une vision intégratrice d'un manager -leader et soulignent un intérêt grandissant pour le leadership (Kanter, 1989 ; Tengblad, 2006). La communication devient plus importante à travers un intérêt grandissant sur le dialogue (Tengblad, 2006), le développement de compétences de négociation (Kanter, 1989) et une augmentation des réunions et des échanges 
informels (Hales, 2001). Le contenu des activités se modifie et la charge de travail augmente tandis que le travail administratif et la fragmentation entre différentes tâches diminuent (Tengblad, 2006). Detchessahar $(2011,2013)$ souligne également l'empêchement des managers qui seraient happés en dehors de la scène du travail pour s'adonner à deux grands types d'activités, à savoir, l'alimentation des systèmes de gestion et le travail en réunion.

Hales (2002) et Tengblad (2006) nuancent le passage d'une bureaucratie vers une post-bureaucratie en indiquant qu'une certaine forme de bureaucratie est toujours présente dans les nouvelles organisations avec une hiérarchie et un degré de formalisation moins élevés et qu'il n'y a pas de rupture radicale dans le métier de manager. Dès lors, les rôles de surveillance des processus de travail, de contrôle des employés et de traitement des informations subsistent aux côtés des nouveaux. Certains auteurs ont étudié le cas des managers intermédiaires (Bellini, 2005 ; Floyd \& Wooldridge, 1994 ; Rouleau, 2005), dont la position « entre le marteau et l'enclume » est particulière (Bellini, 2005) et se révèle critique lors de projets de changement puisqu'ils sont chargés d'opérationnaliser sur le terrain les changements décidés par la sphère stratégique (Balogun \& Johnson, 2004) et donner du sens à ces projets de transformation (Guilmot \& Vas, 2012). Ils s'engagent alors dans un processus de traduction et de création de sens afin de créer une nouvelle vision partagée de la réalité (Balogun \& Johnson, 2004 ; Floyd \& Wooldridge, 1994 ; Rouleau, 2005). Cela les soumet à des attentes de rôles venant à la fois de la hiérarchie et du terrain pouvant parfois se révéler contradictoires et entrainer des conflits de rôles. De par cette position intermédiaire, les recherches pointent des transformations affectant cette catégorie de managers. Par exemple, des rôles stratégiques de traduction et d'agent de changement leur sont reconnus (Balogun, 2003 ; Currie \& Procter, 2005 ; Desmarais \& Abord de Chatillon, 2009; Floyd \& Wooldridge, 1994). Selon Balogun (2003) et Dejoux et Léon (2018), les managers intermédiaires sont davantage sollicités dans un contexte de décentralisation des décisions et d'impératif d'agilité qui les amènent soit à disparaître dans les restructurations, soit à diriger des équipes plus grandes (Balogun, 2003) ou des entités plus petites et décentralisées (Hales, 2001).

Toutefois, la définition du terme « manager » n'est pas consensuelle. Dans cette recherche, nous nous appuyons sur la définition de Payre et Scouarnec (2015, p. 9) qui définissent le manager comme « toute personne qui exerce une fonction d'encadrement, quel que soit son positionnement hiérarchique, fonctionnel ou divisionnel (ou son absence) ainsi que son statut au sein de l'entreprise; auprès d'une ou plusieurs personnes qui exercent ou non, à titre principal, elles-mêmes, une fonction d'encadrement».

\subsection{L'expérience du management}

Afin d'enrichir cette caractérisation théorique, des entretiens exploratoires ont été réalisés avec des managers expérimentés. En faisant varier le genre, les années d'expérience de management, ainsi que le secteur d'activité, nous 


\section{Le métier de manager en transformation: une démarche prospective}

avons constitué un échantillon aléatoire simple et diversifié (Royer \& Zarlowski, 2001) dans le but d'une part, d'éviter les biais liés à une activité-type ou à la personnalité du manager (e.g. Melnik \& Guillemot, 2010 ; Mintzberg, 1984) et, d'autre part, pour appréhender le métier de manager de manière plus holistique. Si le questionnement est similaire à celui qui a animé la réalisation de la revue de la littérature, il s'agit ici de faire émerger, à partir d'acteurs du terrain, les réponses aux questions que nous nous posons concernant l'évolution du métier de manager. La démarche est donc ici davantage inductive en ce qu'elle implique que les chercheurs collectent les données sans a priori structurants qui guideraient les réponses des personnes interviewées (Van Campenhoudt, Marquet \& Quivy, 2017). Cinq entretiens semi-directifs, d'une durée moyenne de 70 minutes, ont été réalisés par deux chercheurs n'ayant pas travaillé sur la revue de la littérature afin d'éviter les a priori structurants, durant les mois d'octobre et de novembre 2016 (cf. Tableau 1).

\section{Tableau 1 - Entretiens exploratoires}

\begin{tabular}{|l|l|l|l|l|}
\hline & $\begin{array}{l}\text { Ancienneté } \\
\text { du répon- } \\
\text { dant dans } \\
\text { la fonction } \\
\text { managériale }\end{array}$ & $\begin{array}{l}\text { Date de } \\
\text { l'entretien }\end{array}$ & $\begin{array}{l}\text { Durée de } \\
\text { l'entretien }\end{array}$ & Secteur d'activité \\
\hline Entretien 1 & 30 ans & $26 / 10 / 2016$ & $62^{\prime} 10^{\prime \prime}$ & Service public - Média \\
\hline Entretien 2 & 25 ans & $15 / 11 / 2016$ & $88^{\prime} 42^{\prime \prime}$ & Banque \& assurances \\
\hline Entretien 3 & 30 ans & $17 / 11 / 2016$ & $87^{\prime} 30^{\prime \prime}$ & Service public - Santé \\
\hline Entretien 4 & 8 ans & $21 / 11 / 2016$ & $29^{\prime} 27^{\prime \prime}$ & Grande distribution \\
\hline Entretien 5 & 10 ans & $23 / 11 / 2016$ & $76^{\prime} 49^{\prime \prime}$ & Banque \& assurances \\
\hline
\end{tabular}

Le guide d'entretien était constitué de huit questions ouvertes dont une question d'ouverture permettant de situer l'acteur dans son contexte et une question de clôture nous assurant, avec la personne interviewée, d'avoir traité tous les éléments importants relatifs à notre question de recherche (Van Campenhoudt et al., 2017). Deux autres questions cherchaient à caractériser le métier actuel de manager. L'objectif premier était d'aborder des éléments issus de la revue de la littérature tels que les activités et le contenu du métier (ex. planification, organisation, coordination, contrôle) (Fayol, 1916 ; Hales, 2001 ; Korica et al., 2015 ; Robbins et al., 2011) ainsi que le rôle du manager (ex. autorité, représentant, décideur) (Mintzberg, 1984). Sur cette base, trois autres questions ont été posées afin d'identifier ( $a$ et b) dans quelle mesure le métier exercé aujourd'hui diffère de celui exercé dans le passé et, (c) comment les répondants perçoivent l'évolution du métier (ex. communication, décentralisation et responsabilisation, sens) (Dejoux \& Léon, 2018 ; Holtz, 2017 ; Lacan, 2016 ; Melnik \& Guillemot, 2010 ; Taskin \& Dietrich, 2016; Tengblad, 2006). Dans le prolongement de ceci, une dernière question visait à identifier les activités et

7. Cette ancienneté implique une activité effective de management d'équipe. 
rôles perçus comme stimulants ou, au contraire, pénibles dans la réalisation du métier de manager. Le second objectif visait à adopter un degré d'ouverture quant aux éléments abordés dans l'entretien et non traités dans la littérature sur le sujet.

Les entretiens ont tous été enregistrés avec l'accord des participants et intégralement retranscrits. Les données collectées ont été traitées par le biais d'une analyse thématique consistant à réduire les données à des thèmes qui sont, pour la plupart, issus du guide d'entretien et des principaux éléments donnés par les répondants (Paillé \& Mucchielli, 2016). Trois thèmes principaux et douze sous-thèmes ont été retenus : (i) les savoir-faire (i.e. gérer des personnes et des équipes, gérer des processus et des projets, former et décider), (ii) les savoir-être (i.e. responsabiliser, se former, être un exemple, être un expert opérationnel, assumer un rôle stratégique), et (iii) les savoirs (i.e. gestion du changement, gestion des diversités, gestion du bien-être).

En termes de savoir-faire, la dimension "gérer des personnes et des équipes » semble centrale dans la caractérisation actuelle du métier du manager. Elle recouvre deux réalités dans le métier de manager. D'une part, il s'agit de gérer une équipe, un collectif et d'autre part, le manager gère des personnes, des individus. Les savoir-faire nécessaires seraient l'écoute, l'évaluation, la gestion des conflits, le coaching ou la reconnaissance, par exemple. De plus, l'analyse des entretiens met en évidence l'importance de la gestion de projets (multiples). Celle-ci mobilise un nombre important de savoir-faire, parmi lesquels la planification ou encore l'anticipation. Le projet est également valorisé comme activité centrale car il renvoie à une expertise générique de gestion (i.e. gérer des processus, des contenus et des personnes) en lien avec une expertise opérationnelle très valorisée par les managers rencontrés (experts dans un domaine particulier, autre que celui du management). De cette expertise, les managers tirent une part importante de leur légitimité. Ces résultats sont cohérents avec les observations de Dumas et Ruiller (2013) concernant le métier de manager dans le secteur hospitalier. Par ailleurs, un savoir-faire supplémentaire souligné est l'organisation du travail (de l'équipe) en observant un principe d'équité. La formation apparaît également comme un élément important dans la caractérisation du métier de manager ainsi que la prise de décision. Souvent associée à sa légitimité, voire à son statut formel, la capacité (voire l'obligation) de décider est un autre aspect important lié au métier de manager.

Aux côtés d'activités clairement pratiquées et commentées, nous avons trouvé d'autres éléments caractéristiques du métier de manager, davantage relatifs à une manière d'être. Il s'agit d'attitudes visant tantôt à accompagner ou responsabiliser, tantôt à insuffler une dynamique, à incarner un modèle, en se formant soi-même par exemple. Ainsi, la délégation et la responsabilisation des collaborateurs seraient également un rôle important du manager qui, ce faisant, exerce la confiance si essentielle à des relations de travail harmonieuses. En corollaire de l'activité de formation des autres, le manager estime important 


\section{Le métier de manager en transformation: une démarche prospective}

de se former lui-même de manière continue au management, mais aussi dans le champ de son expertise professionnelle.

Par ailleurs, une caractéristique supplémentaire porte sur la capacité à interagir avec la direction ou au sein des comités de direction pour représenter son entité mais aussi influencer les décisions et les projets en cours.

D’autres éléments, regroupés sous la bannière des «savoirs » ont été identifiés pour caractériser le métier de manager. Il s'agit de connaissances, qui semblent devoir être acquises et maîtrisées par les managers : la gestion $\mathrm{du}$ changement, d'abord, la gestion des diversités et du bien-être, ensuite. La revue de la littérature et les entretiens exploratoires ont permis d'arrêter une grille de compétences du métier de manager aujourd'hui (Tableau 2).

\section{Tableau 2 - Grille de compétences du métier de manager aujourd'hui}

\begin{tabular}{|c|c|c|}
\hline $\begin{array}{l}\text { Savoir-faire } \\
\text { (manières d'agir) }\end{array}$ & $\begin{array}{l}\text { Savoir-être } \\
\text { (manières d'être) }\end{array}$ & Savoirs \\
\hline $\begin{array}{l}\text { Gérer une équipe et des } \\
\text { personnes }\end{array}$ & Être un exemple & $\begin{array}{l}\text { Avoir une ex- } \\
\text { périence } \\
\text { opérationnelle }\end{array}$ \\
\hline $\begin{array}{l}\text { Gérer des processus et des } \\
\text { projets }\end{array}$ & Être un guide (un leader) & \multirow[t]{6}{*}{$\begin{array}{l}\text { Avoir une expertise } \\
\text { managériale }\end{array}$} \\
\hline Se former & Être responsable & \\
\hline Décider & Être un coach & \\
\hline \multirow[t]{3}{*}{ Communiquer } & Être une figure d'autorité & \\
\hline & Être polyvalent & \\
\hline & Être agile & \\
\hline
\end{tabular}

\section{Etude prospective}

À l'heure actuelle, peu d'études prospectives proposent une caractérisation du métier de manager, demain. Dans la littérature francophone, deux études font office de référence. D'abord, Payre et Scouarnec (2015) proposent une lecture rétro-prospective du métier de manager dans le secteur de la communication publicitaire. Leurs travaux les amènent à identifier cinq scénarios prospectifs sur ce métier, allant du manager stratège au manager mature. Ces auteurs suggèrent que «ce métier est ou va devenir [...] de plus en plus incontournable » et invitent à mieux le définir (Payre \& Scouarnec, 2015, p. 15). Un appel auquel cet article propose de répondre au travers d'une approche centrée sur les compétences et les tensions. Ensuite, Dejoux (2016) identifie trois catégories de compétences qui sont nécessaires pour le manager de demain : des compétences interculturelles, des compétences digitales et des compétences 
d'innovation. Elle avance également que le manager de demain s'orientera vers quatre tendances qui sont le risk manager, le manager agile, le manager stratège et le manager hypermoderne (Dejoux, 2016). Cet article s'inscrit dans le prolongement de ces propositions en cherchant à définir l'ensemble des compétences et nous amène à identifier les tensions qui sont susceptibles de caractériser le métier de manager demain.

\subsection{Démarche prospective}

Selon Jouvenel (2004), la démarche prospective se caractérise par (i) la considération d'un temps long (dans notre cas, l'horizon est l'année 2030), ancrée dans le passé (dans notre cas, la première partie de cette recherche puise la caractérisation du métier de manager jusqu'au début du XXe siècle) ; (ii) l'intégration de ruptures; (iii) une approche pluridisciplinaire systémique (dans notre cas, nous empruntons à la gestion et à la théorie des organisations, considérant que le métier de manager fait partie d'un ensemble plus large, celui de l'organisation et de la société). L'approche prospective est donc holistique et propose la construction, souvent en forme d'arborescence, des futurs possibles (Jouvenel, 2004). D'après Boyer et Scouarnec (2009, p. 89), la prospective métier permet d'identifier « les possibles savoirs et qualifications, expertises ou savoir-faire professionnel, comportements et savoir-être, qui seront demain les plus à même de servir l'individu et l'organisation. Elle nécessite pour cela une coconstruction par les acteurs-experts du métier analysé du devenir possible». Dans cette recherche, nous n'avons pas construit d'arborescence de choix multiples, mais plutôt considéré un seul scénario possible de profil-métier pour le manager de demain, ce qui nous a permis de mettre en évidence une série de tensions inhérentes à l'exercice de ce métier.

\subsubsection{Focus groups}

Afin d'appréhender les transformations futures du métier de manager, nous avons organisé des focus groups regroupant (i) des managers, (ii) des travailleurs et (iii) des PDG, DRH et experts RH (cf. Tableau 3). 
Le métier de manager en transformation: une démarche prospective

\section{Tableau 3 - Présentation des participants aux focus groups}

\begin{tabular}{|c|c|c|c|c|c|}
\hline Date & Profil & Fonction & Sexe & Secteur & Label \\
\hline \multirow{3}{*}{ 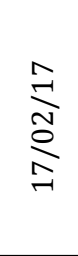 } & \multirow{3}{*}{ 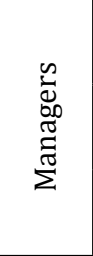 } & $\begin{array}{l}\text { Responsable du changement et } \\
\text { de la communication (NFOT) }\end{array}$ & $F$ & \begin{tabular}{|l|} 
Privé / \\
Assurances
\end{tabular} & Manager 1 \\
\hline & & Directeur d'un bureau régional & $\mathrm{H}$ & $\begin{array}{l}\text { Public / } \\
\text { Santé }\end{array}$ & Manager 2 \\
\hline & & $\begin{array}{l}\text { Directeur du commerce et du } \\
\text { marché public }\end{array}$ & $\mathrm{H}$ & \begin{tabular}{|l|} 
Privé / \\
Énergie \\
\end{tabular} & Manager 3 \\
\hline \multirow{7}{*}{ 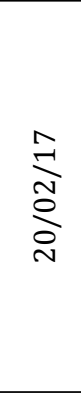 } & \multirow{7}{*}{ 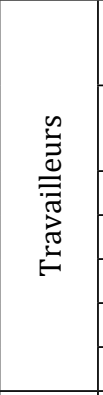 } & $\begin{array}{l}\text { Directeur du secteur et du } \\
\text { contenu du management }\end{array}$ & $\mathrm{H}$ & $\begin{array}{l}\text { Privé / } \\
\text { Banque }\end{array}$ & Travailleur 1 \\
\hline & & Responsable E-communication & $\mathrm{H}$ & $\begin{array}{l}\text { Privé / } \\
\text { Industrie }\end{array}$ & Travailleur 2 \\
\hline & & Analyste business & $\mathrm{F}$ & Privé / IT & Travailleur 3 \\
\hline & & Ingénieur logiciel & $\mathrm{H}$ & Privé / IT & Travailleur 4 \\
\hline & & Conseiller en développement & $\mathrm{F}$ & Public / RH & Travailleur 5 \\
\hline & & Conseiller Général & $\mathrm{H}$ & Public / RH & Travailleur 6 \\
\hline & & Reconversion professionnelle & $\mathrm{F}$ & Privé / RH & Travailleur 7 \\
\hline \multirow{6}{*}{ 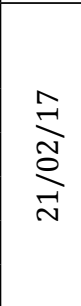 } & \multirow{6}{*}{ 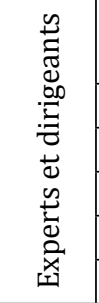 } & $\begin{array}{l}\text { Maitre de conférences \& } \\
\text { consultant }\end{array}$ & $\mathrm{H}$ & Privé / RH & Expert 1 \\
\hline & & PDG & $\mathrm{H}$ & Privé / IT & Dirigeant 1 \\
\hline & & PDG & $\mathrm{H}$ & Privé / RH & Dirigeant 2 \\
\hline & & Consultante RH & $\mathrm{F}$ & Privé / RH & Expert 2 \\
\hline & & DRH d'un Service Public & $\mathrm{F}$ & Public / RH & Expert 3 \\
\hline & & PDG & $\mathrm{H}$ & Privé / IT & Dirigeant 3 \\
\hline
\end{tabular}

Ces focus groups ont eu lieu entre le 17 et le 21 février 2017. Ils ont duré chacun en moyenne 2 h 30 et ont été enregistrés avec l'accord des participants et intégralement retranscrits. Chaque focus group s'est déroulé en trois étapes : (i) une réflexion collective sur la manière dont les participants voient le métier de manager en 2030, (ii) une réflexion sur les compétences associées au métier de manager en lien avec leur vision, et (iii) une réflexion sur base du référentiel de compétences du manager «aujourd'hui » afin d'évaluer et souligner les compétences amenées à se développer et à disparaître.

L'analyse des retranscriptions a permis l'émergence de thèmes dans une démarche inductive et collective qui s'apparente à une analyse phénoménologique interprétative (Frost et al., 2010 ; Smith, 2004). Concrètement, nous avons sélectionné des verbatim (i.e. des citations textuelles tirées des entretiens) et les avons regroupés en fonction du thème correspondant le mieux à l'argument du participant. Ensuite, nous les avons organisés en thèmes et sous-thèmes (Tableau 4). 


\section{Tableau 4 - Thèmes et sous-thèmes}

\begin{tabular}{|c|c|c|}
\hline Thèmes & Sous-thèmes & Définitions \\
\hline \multirow{5}{*}{$\begin{array}{l}\text { Gestion des } \\
\text { équipes } \\
\text { et des } \\
\text { personnes }\end{array}$} & Développement & $\begin{array}{l}\text { Capacité à gérer le développement des } \\
\text { personnes et à les motiver en veillant au } \\
\text { respect de l'équilibre vie privée - vie pro- } \\
\text { fessionnelle et de leurs perspectives de } \\
\text { carrière et de développement }\end{array}$ \\
\hline & Sens et valeurs & $\begin{array}{l}\text { Connaissance et transmission du sens du } \\
\text { travail et des valeurs, missions et de la } \\
\text { vision d'entreprise }\end{array}$ \\
\hline & Gestion de la diversité & $\begin{array}{l}\text { Capacité à respecter, à s'adapter à } \\
\text { et à gérer la diversité culturelle et } \\
\text { générationnelle }\end{array}$ \\
\hline & $\begin{array}{l}\text { Facilitateur/ } \\
\text { animateur }\end{array}$ & $\begin{array}{l}\text { Capacité à animer un collectif de travail } \\
\text { pouvant être partiellement déspatialisé } \\
\text { et en facilitant les échanges par le biais de } \\
\text { différents canaux de communication }\end{array}$ \\
\hline & Référent/coach & $\begin{array}{l}\text { Capacité à responsabiliser et à guider les } \\
\text { personnes dans l'atteinte de leurs objec- } \\
\text { tifs tout en restant un référent disponible }\end{array}$ \\
\hline $\begin{array}{l}\text { Gestion des } \\
\text { processus de } \\
\text { travail }\end{array}$ & $\begin{array}{l}\text { Organisation du travail } \\
\text { Vue hélicoptère/ } \\
\text { transversale } \\
\end{array}$ & $\begin{array}{l}\text { Capacité à coordonner le travail } \\
\text { intra-équipes et inter-équipes }\end{array}$ \\
\hline Polyvalence & & $\begin{array}{l}\text { Capacité à s'adapter aux besoins organi- } \\
\text { sationnels en déployant les compétences } \\
\text { transversales nécessaires au passage } \\
\text { d'une équipe à l'autre, d'un projet à l'autre }\end{array}$ \\
\hline $\begin{array}{l}\text { Gestion du } \\
\text { changement }\end{array}$ & & $\begin{array}{l}\text { Capacité à identifier (voire à anticiper) un } \\
\text { changement affectant l'organisation et/ou } \\
\text { son contexte et à accompagner les adapta- } \\
\text { tions nécessaires }\end{array}$ \\
\hline
\end{tabular}

\subsection{Résultats}

Les résultats de notre analyse empirique sur le métier de manager en 2030 tel qu'envisagé par les managers, les travailleurs et les DRH, PDG et experts que nous avons rencontrés, permettent d'identifier quatre dimensions caractérisant ce métier : (i) la gestion des équipes et des personnes, (ii) la gestion des processus de travail, (iii) la gestion du changement, et (iv) la polyvalence. Les participants de la recherche ont particulièrement insisté sur la dimension «gestion des équipes et des personnes » comme étant un aspect central dans le profil du manager à l'horizon 2030. 


\section{Le métier de manager en transformation : une démarche prospective}

\subsubsection{Gérer des équipes et des personnes}

Déjà centrale dans la caractérisation du métier de manager aujourd'hui, la dimension "gestion d'équipe et des personnes » semble résister au temps et prendre une importance significative, dans une visée prospective. Le métier de manager serait ainsi amené à se professionnaliser davantage et à se transformer en celui de gestionnaire des équipes et des personnes, qui consiste à gérer des individus et à animer un collectif. De fait, les répondants estiment que répondre aux différents besoins et attentes des collaborateurs par rapport à leur travail, constituerait une facette importante du manager à l'avenir. À cet égard, la capacité d'identifier et de gérer la satisfaction des besoins individuels constituerait une compétence managériale cruciale. À titre d'exemple, plusieurs répondants mettent en avant la nécessité grandissante de prendre en considération la qualité de vie, dans et en dehors du travail, qui se traduirait notamment par une plus grande souplesse dans l'articulation entre les temps de vie et de travail.

Si le développement des personnes fait référence à l'épanouissement personnel par le travail, il affère également à la gestion des carrières. Demain, les aspirations professionnelles individuelles nécessiteraient une prise en considération plus importante de la part du manager. De cette manière, les répondants se représentent le manager de demain comme une personne en charge d'anticiper, avec le travailleur, les prochaines étapes du développement de sa carrière.

«Je crois personnellement que les entreprises qui auront le plus de succès en 2030 sont celles qui auront géré le mieux la nouvelle génération, qui permettent à la fois d'attirer les plus gros talents, et ce ne sera pas avec les plus beaux salaires, mais bien avec le plus bel équilibre vie privée - vie professionnelle ; répondre à leurs aspirations. » (Manager 3).

Si ce scénario visant à répondre aux aspirations professionnelles se confirme, les managers pourraient aussi être face à des travailleurs de plus en plus nomades. Dans ce contexte, l'écoute des collaborateurs en vue de mieux les orienter, les conseiller, les guider et les accompagner afin qu'ils se développent constituerait une autre compétence fondamentale du manager de demain. De même, la gestion de la diversité pourrait devenir une compétence importante du manager de demain.

Dans le prolongement du développement des personnes émerge la question du sens au travail et par le travail. Selon les répondants, la capacité du manager à transmettre le sens des activités réalisées par l'équipe travail, en expliquant et en partageant les informations, dans un contexte perçu comme cohérent où chacun trouve sa place, prendrait une place prépondérante à l'avenir. Audelà du sens, il serait attendu du manager de demain qu'il soit l'ambassadeur des valeurs de l'entreprise, désormais davantage orientées vers l'éthique et la durabilité, sous l'effet croissant de la responsabilité sociétale des entreprises. 
"Quelqu'un qui, connaissant la vision d'une organisation, etc., et vers où elle se projette, est capable de créer du sens, est capable à ce moment-là aussi, de mobiliser des ressources. » (Expert 1).

Les personnes interrogées envisagent finalement le manager de demain comme l'animateur d'un collectif, comme un facilitateur à qui il incomberait d'ouvrir des espaces de discussion mais également d'intervenir lorsque la situation est bloquée. Autrement dit, être au service de son équipe et accompagner ses membres dans les décisions qu'ils prennent de manière autonome et collective. En ce sens, la mise en place des systèmes de communication facilitant la transmission des messages - notamment dans la situation d'un management à distance - deviendrait une compétence cruciale pour le manager de demain.

Le manager de demain pourrait être résumé à l'image d'un coach ou d'un coordinateur équipé de solides softs skills. En phase avec les observations de Theurelle-Stein et Barth (2017) concernant l'importance des compétences «soft» dans le métier de manager, nos répondants ont insisté sur l'importance de l'écoute, le partage d'information, la délégation, l'accompagnement, le soutien, etc., autant d'éléments qui caractériseraient les compétences du coach mais qui renforceraient également l'idée selon laquelle le manager de 2030 serait aussi une personne ressource, un référent, disponible pour ses collaborateurs. En effet, la disponibilité est apparue comme un enjeu amené à s'intensifier avec le développement des nouvelles technologies de l'information et de la communication.

"Une connaissance de l'humain. Comment fonctionne un humain en tant qu'individu, et en société, en collectif (...). Pour moi, il y a une dimension soft skills qui devient nécessaire, une science molle, qui devient une nécessité pour pouvoir accompagner les personnes» (Dirigeant 1).

Cette dimension de gestionnaire des équipes et des personnes met en lumière deux tensions inhérentes au métier de manager à l'avenir. Premièrement, entre la gestion des niveaux individuel et collectif. Si ce scénario venait à se confirmer, le manager de demain devrait davantage prendre en compte les besoins individuels des membres de son/ses équipe(s) tout en étant garant et animateur du collectif de travail. Ce constat questionne la capacité du manager de demain à assurer cet équilibre entre les niveaux individuel et collectif.

Deuxièmement, entre la responsabilité du manager quant aux résultats du travail de l'équipe et celle des individus. Bien que la responsabilisation des travailleurs et le partage des prises de décision soient identifiés comme une tendance pour le futur, peu s'accordent sur l'identification de la responsabilité finale par rapport au travail, aux résultats. En filigrane, c'est la (re)configuration de l'autorité qui est aussi questionnée ici. 


\section{Le métier de manager en transformation : une démarche prospective}

\subsubsection{Gérer des processus de travail}

Comme souligné auparavant, à l'horizon 2030, les travailleurs seraient davantage responsables. Dans ce cadre, le manager serait amené à délaisser certains aspects liés à l'organisation du travail pour se concentrer davantage sur le coaching des personnes ainsi que le cadrage du déroulement des activités. D'ailleurs, il déléguerait certaines tâches traditionnellement associées au management quand cela sera jugé nécessaire (e.g. tâches administratives, reporting, etc.). Néanmoins, les répondants estiment que le manager de demain sera capable d'avoir une "vue hélicoptère » sur les différentes activités, les divers projets en cours dans l'organisation. Cela implique un large éventail de compétences transversales lui permettant de saisir, du moins superficiellement, les enjeux des différents départements et activités associées. Dans cette perspective, organiser le travail différemment, utiliser de nouvelles méthodes de gestion de projets ou de nouveaux processus de travail, s'avéreraient nécessaires. En tant que coordinateur, le manager devra être capable de mobiliser les ressources nécessaires à son/ses projets, faire du réseautage, se charger des achats, etc.

«Il essaye quand même de garder l'église au milieu du village, parce qu'on est peut-être focalisé sur une partie de son boulot, [alors que] l'équipe d'à-côté travaille sur un projet, il y a peut-être des interconnexions qui doivent se faire. Et je pense que lui, il doit être un peu au-dessus de la mêlée pour voir si l'équipe d'à-côté n'est pas en train de faire la même chose que lui, ou si on n'aurait pas intérêt à agir ensemble. » (Travailleur 2)

\subsubsection{Gérer le changement}

Si une chose devait rester stable à l'avenir, c'est le changement, estiment les répondants. En ce sens, l'importance des compétences de gestion du changement du manager de demain serait cruciale. D'autant plus que le manager aura à faire face à des changements de différentes natures à l'horizon 2030 liés à des tendances telles que la digitalisation des activités, la croissance démographique, la transformation de la mobilité, etc. Le manager, dans ce contexte, serait amené à s'adapter constamment à ce nouveau contexte, ainsi qu'à gérer les effets de ces changements sur le travail. Par ailleurs, la stabilité temporelle des équipes de travail serait amenée à diminuer dans le futur.

"Je ne pense pas que ça existera encore dans le futur : les équipes dans lesquelles on travaille depuis plus de 10 ans, je pense que ça va disparaître. Donc oui, être beaucoup plus flexible et changer d'équipe et de contenu de plus en plus. » (Manager 1).

\subsubsection{Etre polyvalent}

Si la nature et le contenu de son métier amène le manager d'aujourd'hui à se rendre polyvalent, d'après nos répondants, il semblerait que le manager de 2030 ait à s'adapter régulièrement à des nouvelles équipes, à des nouveaux projets et par conséquent, à des contenus différents au niveau du travail. Si ce 
scénario semble plausible et acceptable aujourd'hui, il marque toutefois une réelle évolution du métier de manager. En effet, par le passé, il était commun de voir un manager gérer une équipe composée des mêmes personnes et travaillant sur les mêmes tâches pendant plusieurs années consécutives. Selon nos répondants toujours, les équipes de travail seront plus nombreuses, plus souples et temporaires et le manager devrait être capable de passer de l'un(e) à l'autre selon les besoins de l'organisation du travail. Cette idée d'une polyvalence croissante dans le chef des managers est notamment illustrée dans le verbatim précédent.

En outre, de par cette polyvalence croissante et caractérisant sa fonction à l'avenir, il semblerait que le manager ait à se tenir de plus en plus informé de ce qui se fait ailleurs, tant dans d'autres services au sein de son organisation qu'en dehors de celle-ci, comme l'illustre le verbatim suivant.

"Il ne faut pas savoir uniquement ce qu'on fait dans son équipe mais il faut savoir aussi ce que les collègues font à côté. Parce que souvent, il y avait des projets quasi similaires qui se développaient dans deux endroits différents, surtout quand il s'agit de sièges différents. » (Manager 2).

Ceci suggère donc que le manager devra être amené de plus en plus à sortir de sa zone d'attribution, et devra donc certainement apprendre à gérer de manière flexible les frontières entre ses projets, ses équipes, ses compétences, et l'ensemble des éléments périphériques avec lesquels il devra travailler au quotidien.

Cette dimension du métier de manager soulève également une troisième tension à venir dans l'exercice de ce métier : si le contenu du métier de manager est amené à se diversifier (par le biais d'une plus grande polyvalence), plusieurs questions se posent, notamment concernant la légitimité du métier de manager. En effet, les CEO, DRH, experts et managers rencontrés s'accordent pour reconnaître que la gestion des équipes, des personnes, de l'organisation $\mathrm{du}$ travail et des processus de gestion constitue un métier à part entière, et qu'il ne serait plus, à l'avenir, compatible avec l'exercice de responsabilités opérationnelles. Autrement dit, le manager de demain sera un manager, et plus un expert combinant son travail d'expert avec la responsabilité de la gestion d'une équipe ou d'une entité. Les salariés rencontrés sont, eux, davantage attachés à pouvoir trouver, auprès de leur manager, un expert des domaines d'activités qu'ils exercent. Cette question est d'autant plus cruciale dans un contexte où l'innovation est rapide. En effet, si cela se confirme, le manager ne pourrait plus capitaliser sur son expertise passée et devrait donc se maintenir à jour, mais la question quant aux moyens et ressources mises à sa disposition pour qu'il y parvienne reste en suspens. En outre, dans le cas où le contenu du métier de manager porterait exclusivement sur les aspects de gestion des personnes, des équipes et des processus d'organisation du travail, c'est aussi la plus-value apportée par les métiers de la GRH qui se trouve questionnée. 


\section{Le métier de manager en transformation:}

une démarche prospective

À l'issue de la présentation des résultats des contours du métier de manager aujourd'hui et demain, au travers de l'étude prospective qui a été menée, des tensions ont été identifiées et permettent d'envisager les défis du métier de manager de demain.

\subsection{L'apport d'une perspective qui décrit le métier de manager au regard des tensions qui le traversent}

La démarche prospective adoptée dans cette recherche apparaît comme complémentaire de la démarche rétro-prospective de Payre et Scouarnec (2015) ayant contribué à l'identification de cinq scénarios dans le secteur spécifique de la communication publicitaire. En particulier, le portrait du manager décrit par nos résultats fait écho à trois des scénarios identifiés par Payre et Scouarnec (2015), à savoir les scénarios du manager 2.0, du manager de projets, et du manager mature. Nos résultats amènent donc à considérer que ces scénarios ne sont pas nécessairement mutuellement exclusifs.

Outre présenter un profil prospectif, le choix méthodologique issu de nos analyses qualitatives nous a amené à identifier trois tensions majeures inhérentes à l'exercice du métier de manager demain. D'abord, une tension relative à la combinaison des niveaux individuels (tenir compte des aspirations de chaque individu, de ses contraintes, de sa personnalité...) et collectif (là où le travail donne du sens, où une communauté doit exister, où la collaboration doit s'exercer...) dans l'action de gérer. Dans la prévision esquissée par nos répondants, tant les managers expérimentés qu'à l'occasion des focus groups, il ressort qu'il faudra demain une gestion qui soit plus individualisée tout en portant aussi sur l'affirmation plus marquée de la dimension collective, de la communauté de travail d'où le sens du travail doit aussi émerger. Cette première tension rappelle les constats de Dietrich (2009, p. 202) qui affirme que gérer « la cohésion d'un collectif et « distinguer» des individus peuvent relever de logiques contradictoires ». Ensuite, une tension relative à la responsabilité du manager dans un contexte de travail qui semble, demain, davantage responsabiliser les individus mis en réseaux (tribus, chapitres, cercles et autres notions utilisées pour caractériser des groupes de travail) quant à leurs actions (autonomie) et résultats (responsabilité), mais aussi dans lequel le manager, par les compétences plus vastes qui lui sont reconnues, notamment en termes d'accompagnement des individus et des groupes, garde la responsabilité de l'animation des équipes, voire de leur évaluation. La tension tient ici de la limite jusqu'à laquelle la fonction d'autorité qu'incarne le manager est partagée. Enfin, une tension relative au positionnement du manager par rapport à une expertise « autre ». Il s'agit ici de reconnaître (ou pas) que le métier de manager est un métier en tant que tel et qu'il se suffit à lui-même : pour gérer une équipe de juristes, pas besoin d'être juriste soi-même, finalement. Si cette perspective est celle partagée par les PDG, DRH, experts et managers rencontrés dans le cadre de l'étude prospective, elle n'est pas tant partagée par les travailleurs. Eux attendent de leur manager qu'il soit aussi un référent fonctionnel. La 
distinction des liens hiérarchique et fonctionnel, pourtant effective dans de nombreuses organisations aujourd'hui, demeure une tension pour le métier de manager, demain.

C'est parce que ces tensions sont apparues concomitamment à l'analyse de nos résultats et, singulièrement, à l'identification des quatre composantes essentielles à l'exercice du métier de manager demain (la gestion des équipes et des personnes, des processus de travail, du changement et la capacité à être polyvalent) que nous avons considéré un seul scénario prévisionnel pour le métier de manager demain. Cette situation unique est donc plus nuancée que son découpage en scénarios multiples, tranchant chaque point de tension en situations différentes allant de la résolution totale de chacune de ces tensions dans un sens puis dans l'autre. Ainsi, le métier de manager apparaît en tensions, ce qui semble correspondre aux témoignages recueillis. Celles-ci s'expriment et se gèrent différemment en fonction des contextes organisationnels (structure, culture...) et des acteurs en présence et la façon de les gérer. Les équilibres qui seront aménagés définissent des contours différents du métier de manager, demain.

\section{Discussion}

\subsection{Les implications de cette caractérisation du métier en termes d'accompagnement $\mathrm{RH}$ et de formation au métier du manager}

Cette évolution possible du métier de manager appelle certains accompagnements RH. Parmi ceux-ci, et à l'image de ce que préconisent Payre et Scouarnec (2015), les stratégies en matière de GRH pourraient intégrer un axe de développement managérial. Les référentiels présentés dans cet article sont des guides qui peuvent accompagner ces stratégies $\mathrm{RH}$, comme c'est le cas dans quelques organisations auxquelles ce scénario a été présenté. Plus fondamentalement, la prise en main du développement de l'expertise managériale et de sa légitimation au sein des organisations et des services dédiés à la GRH est un axe de développement majeur dans la perspective d'un management humain envisagée par certains comme préfigurant la GRH de demain (e.g. Taskin \& Dietrich, 2016). Il s'agit, ici, de se (re)concentrer sur l'expertise, le métier, le travail réel et vivant du manager - au service des autres expertises de l'organisation - plutôt que de prôner un management du bonheur ou du bien-être qui semble parfois trop générique et davantage ancré dans des narrations enthousiastes.

L'évolution du métier de manager esquissée dans notre étude prospective interroge aussi la formation au management. Si le manque d'adéquation entre la formation au management et l'exercice du métier de manager est régulièrement pointé du doigt (de Woot \& Kleymann, 2011; Dupuy, 2015 ; Mintzberg, 2005), c'est souvent au regard de la technicité des savoirs qui sont enseignés et du 


\section{Le métier de manager en transformation:}

une démarche prospective

caractère fonctionnaliste des connaissances. L'orientation professionnelle de la formation s'explique par l'histoire des grandes écoles (initiées et associées aux chambres de commerce et d'industrie) mais aussi par la proximité des enseignements, des recherches et des besoins des entreprises, voulue tant par les étudiants que les responsables de ces établissements, au nom de l'employabilité. La perspective que nous esquissons ici tranche avec ce type de savoirs techniques univoques basés sur des modèles universels. À l'inverse, le scénario retenu invite à former les managers de demain à la gestion des tensions auxquelles ils seront immanquablement confrontés dans l'exercice de leur métier. Plutôt que de trancher, en cherchant à appliquer le modèle qui permet de résoudre le problème, il s'agit donc de trouver un équilibre entre des dimensions très sensibles pour les travailleurs et les organisations : le sens, la responsabilité et la reconnaissance d'une expertise. C'est ici une invitation pour nos écoles de commerce et les nombreux acteurs de la formation au management, à former à la capacité d'organiser plus qu'à la gestion, tout en montrant que la variété des manières selon lesquelles les individus s'organisent est bien plus importante et répandue que les quelques modèles universels qui sont enseignés (Parker, 2018). Cela fait notamment écho à l'appel de Petriglieri et Petriglieri (2015) à repenser la formation dans les écoles de commerce en adoptant des approches contribuant à la ré-humanisation du leadership, c'està-dire notamment en considérant le leadership comme un processus continu durant lequel un individu devient leader, et accède à la validation de ses pairs dans ce rôle, transcendant par là-même son identité. Alors que les termes de manager et de leader font appel à des définitions différentes, on peut suggérer que la formation au management intègre, à l'avenir, davantage de formation au leadership et à l'apprentissage de l'humain (Cristol, 2009).

Enfin, au-delà de repenser la formation au métier de manager au sein des écoles de commerce, il convient également de repenser la formation continue au service des managers. À ce titre, le mouvement de l'éducation critique en management, découlant des perspectives critiques en gestion, ouvre des perspectives nouvelles de réflexion pour repenser la fonction managériale. C'est le cas notamment des initiatives de formation continue basée sur l'art, et se présentant comme de nouvelles méthodes permettant de repenser de manière créative la fonction managériale, et «à préparer les managers à se confronter à des environnements de plus en plus complexes, dynamiques, chaotiques et subjectifs » (Falcoz \& Livian, 2015, p. 59). Outre les initiatives basées sur l'art, les résultats de Dumazert, Lemoine et Drillon (2010) quant à la fonction de chef de guilde au sein des jeux multi-joueurs suggèrent que la frontière entre le management du réel et le management du virtuel est plutôt ténue. Dès lors, cela amène également à envisager des formes d'apprentissage innovantes pouvant reposer sur le jeu et la virtualité, et constituant autant d'opportunités de tester ses capacités managériales, ou les développer. En conclusion, au-delà de la formation initiale au sein des écoles de gestion, il nous apparaît que repenser la fonction managériale peut également passer par une réflexion quant aux modalités de la formation continue. 


\section{Conclusion}

Le contexte du travail et des modes d'organisation du travail contemporains invite à actualiser les modèles traditionnels décrivant le métier de manager. Cet article répond à cette invitation en caractérisant le métier de manager aujourd'hui au travers d'une grille de compétences (basée sur une revue de la littérature et des entretiens avec des managers expérimentés). Ensuite, une démarche prospective permet de présenter un scénario d'évolution du métier de manager à l'horizon 2030. Ces résultats mettent en exergue un métier en tensions, concernant la responsabilité de la décision, la combinaison d'une expertise gestionnaire et technique, le besoin de gérer des individualités et la nécessité de fédérer un collectif.

\section{Bibliographie}

ADECCO (2017), Digitalisation et robotisation : Réinventer les métiers?, Retrieved from http://www.groupe-adecco.fr/digitalisationrobotisation-reinventer-les-metiers/

BALOGUN J. (2003), "From Blaming the Middle to Harnessing its Potential : Creating Change Intermediaries", British Journal of Management, 14(1), 69-83. http ://doi.org/10.1111/1467-8551.00266.

BALOGUN J. \& JOHNSON G. (2004), “Organizational restructuring and Middle Manager sensemaking”, Academy of Management Journal, 47(4), 523-549.

BARKER R.A. (1997), "How Can We Train Leaders if We Do Not Know What Leadership Is ?", Human Relations, 50(4), 343-362. http://doi. org/10.1177/001872679705000402.

BARLEY S. \& KUNDA G. (2001), “Bringing Work Back In”, Organization Science, 12(1), 76-95.

BELLINI S. (2005), «Éviter le marteau, glisser sur l'enclume. Comment les managers de proximité ajustent leurs rôles face aux contraintes de l'organisation », Les Cahiers Internationaux de Psychologie Sociale, 65(1), 13-25. http ://doi.org/10.3917/cips.065.0013.

BOYER L. \& SCOUARNEC A. (2009), « De la prospective des métiers à la prospective des talents ». In Peretti J.M., Tous talentueux (pp. 87-92). Eyrolles, éditions d'organisation.

CFDT (2017), Parlons Travail : Rapport de l'enquête sur le travail de la CFDT.

CRISTOL D. (2009), « L'enseignement des sciences de gestion s'oppose-t-il à l'apprentissage du management? », Revue Internationale de Psychosociologie, 15, 307-325.

CURRIE G. \& PROCTER S.J. (2005), "The Antecedents of Middle Managers' Strategic Contribution: The Case of a Professional 
Le métier de manager en transformation: une démarche prospective

Bureaucracy", Journal of Management Studies, 42(7), 1325-1356. http ://doi. org/10.1111/j.1467-6486.2005.00546.x

DE WOOT P. \& KLEYMANN B. (2011), «Changer le paradigme culturel du management: Un impératif, pas une option ». In Taskin L. \& de Nanteuil M. (Eds.), Perspectives critiques en Management. Pour une gestion citoyenne, De Boeck, Bruxelles, Belgique.

DEJOUX C. (2016), Du management au leadership agile (2 $2^{\text {nd }}$ ed.), Dunod.

DEJOUX C. \& LÉON E. (2018), Métamorphose des managers à l'ère du numérique et de l'intelligence artificielle ( $5^{\mathrm{e}}$ édition), Pearson.

DESMARAIS C. \& ABORD DE CHATILLON E. (2009), « Les managers et leurs rôles: La traduction, un talent et une ressource». In Peretti J.M., Tous talentueux (pp. 65-71).

DETCHESSAHAR M. (2011), «Santé au travail. Quand le management n'est pas le problème, mais la solution », Revue Française de Gestion, 37(214), 89-105. http ://doi.org/10.3166/rfg.214.89-105.

DETCHESSAHAR M. (2013), «Faire face aux risques psycho-sociaux: quelques éléments d'un management par la discussion », Négociations, 19(1), 57. http ://doi.org/10.3917/neg.019.0057.

DIETRICH A. (2009), « Le manager intermédiaire ou la GRH mise en scène », Management \& Avenir, 1(21), 196-206. http ://doi.org/10.3917/mav.021.0196.

DUMAS M. \& RUILLER C. (2013), «Être cadre de santé de proximité à l'hôpital, quels rôles à tenir? », Revue de Gestion Des Ressources Humaines, 87(1), 42-58. http ://doi.org/10.3917/grhu.087.0042.

DUMAZERT J.-P., LEMOINE L. \& DRILLON D. (2010), « Prospective sectorielle appliquée aux mondes virtuels. De la fonction de chef de guilde au métier de manager. Quelle réflexion à partir des jeux massivement multi-joueurs? ", Management \& Avenir, 36(6), 298-315. http ://doi.org/10.3917/mav.036.0298.

DUMAZERT J. (2011), « Chef de guilde et métier de manager : un constat RH pour une prospective sectorielle appliquée aux mondes virtuels », Management \& Avenir, 9(49), 256-276.

DUMAZERT J. (2017), « Le leader de guilde, un manager réflexif porté par son double virtuel », Revue Internationale de Psychosociologie et de Gestion Des Comportements Organisationnels, Supplément, 113 à 132.

DUPUY F. (2015), La faillite de la pensée managériale, Seuil.

FALCOZ C. \& LIVIAN Y.-F. (2015), « Des managers en formation au Louvre : usages de la peinture dans un cursus de formation continue», Annales Des Mines - Gérer et Comprendre, 120, 58-66.

FAYOL H. (1916), Administration générale et industrielle (6 $6^{\text {th }}$ ed.), Dunod, Paris. 
FLOYD S.W. \& WOOLDRIDGE B. (1994), “Dinosaurs or dynamos ? Recognizing middle management's strategic role”, Academy of Management Executive, 8(4), 47-57. http ://doi.org/10.5465/ame.1994.9412071702.

FROST N., NOLAS S.M., BROOKS-GORDON B., ESIN C., HOLT A., MEHDIZADEH L., \& SHINEBOURNE P. (2010), "Pluralism in qualitative research : the impact of different researchers and qualitative approaches on the analysis of qualitative data", Qualitative Research, 10(4), 441-460. http :// doi.org/10.1177/1468794110366802.

GREY C. \& GARSTEN C. (2001), "Trust, Control and Post-Bureaucracy", Organization Studies, 22(2), 229-250. http://doi.org/10.1177/ 0170840601222003.

GUILMOT N. \& VAS A. (2012), «Les cadres intermediaires au coeur du sensemaking ", Revue Internationale de Psychosociologie et de Gestion Des Comportements Organisationnels, XVIII(45), 77. http://doi.org/10.3917/ rips1.045.0077.

HALES C. (1986), "What do managers do ? A critical review of the evidence", Journal of Management Studies, 23(1), 88-115. http ://doi. org/10.1111/j.1467-6486.1986.tb00936.x.

HALES C. (2001), “What Managers Do ?”, Business Strategy Review, 12(2), 50-58.

HALES C. (2002), "Bureaucracy-lite and Continuities in Managerial Work", British Journal of Management, 13(1), 51-66. http://doi. org/10.1111/1467-8551.00222.

HOLTZ T. (2017), « Mutations du leadership dans une entreprise en voie de libération ", Revue Internationale de Psychosociologie et de Gestion Des Comportements Organisationnels, 23(2), 125-140.

INSTITUTE FOR THE FUTURE \& DELL TECHNOLOGIES (2017), Emerging Technologies' Impact On Society \& Work In 2030. Retrieved from https :// www.delltechnologies.com/content/dam/delltechnologies/assets/perspectives/2030/pdf/SR1940_IFTFforDellTechnologies_Human-Machine_070517_ readerhigh-res.pdf

JOUVENEL H. (2004), Invitation à la prospective - An Invitation to Foresight, Futuribles.

KANTER R. (1989), "The new managerial work”, Harvard Business Review, 67, 85-92.

KNIGHTS D. \& WILLMOTT H. (2007), Introducing organizational behaviour and management, London: Thomson.

KORICA M., NICOLINI D. \& JOHNSON B. (2015), "In Search of 'Managerial Work' : Past, Present and Future of an Analytical Category”, International Journal of Management Reviews, 00, n/a-n/a. http ://doi.org/10.1111/ijmr.12090. 
Le métier de manager en transformation: une démarche prospective

LACAN A. (2016), « La postmodernité dans l'entreprise : quel manager pour relever le défi ? », Management \& Avenir, 90(8), 195. http ://doi.org/10.3917/ mav.090.0195.

MCKINSEY GLOBAL INSTITUTE (2017), Jobs Lost, Jobs Gained : Workforce Transitions In A Time Of Automation, Retrieved from : https ://www.mckinsey. com/ /media/McKinsey/Featured Insights/Future of Organizations/What the future of work will mean for jobs skills and wages/MGI-Jobs-Lost-JobsGained-Report-December-6-2017.ashx

MELNIK E. \& GUILLEMOT D. (2010), «Vers une convergence du management public-privé ? Une revue de littérature économique », Revue Française d'économie, 2(25), 167-225.

MINTZBERG H. (1975), "The manager's job : folklore and fact”, Harvard Business Review, 49-61.

MINTZBERG H. (1984), Le manager au quotidien : les dix rôles du cadre, Les Editions d'Organisation, Paris.

MINTZBERG H. (2005), Des managers, des vrais, pas des MBA : un regard critique sur le management et son enseignement, Editions d'Organisation, Paris.

PAILLÉ P. \& MUCCHIELLI A. (2016), L'analyse qualitative en sciences humaines et sociales ( $\left.4^{\mathrm{e}} \mathrm{ed}\right)$, Armand Colin.

PARKER M. (2018), Shut Down the Business School! An insider's account of what's wrong with management education, Pluto press.

PAYRE S. (2010), Manager les hommes et leurs comportements afin de mieux prendre en charge les problèmes de santé au travail, Nouveaux comportements, nouvelle GRH.

PAYRE S. \& SCOUARNEC A. (2015), « Manager : Un métier en mutation? Essai de lecture rétro-prospective pour dessiner les contours du métier de manager et les accompagnements RH nécessaires ", Revue de Gestion Des Ressources Humaines, 97(3), 3-16. http ://doi.org/10.3917/grhu.097.0003.

PETRIGLIERI G. \& PETRIGLIERI J.-L. (2015), “Can Business Schools Humanize Leadership ?", Academy of Management Learning \& Education, 14(4), 625-647. Retrieved from http ://search.ebscohost.com/login. aspx ?direct $=$ true $\& d b=b t h \& A N=111805002 \&$ site $=$ ehost-live

ROBBINS S., DECENZO D. \& COULTER M. (2011), Management, l'essentiel des concepts et pratiques ( $7^{\text {th }}$ ed.), Pearson.

ROULEAU L. (2005), "Micro-Practices of Strategic Sensemaking and Sensegiving: How Middle Managers Interpret and Sell Change Every Day", Journal of Management Studies, 42(7), 1413-1441. http ://doi. org/10.1111/j.1467-6486.2005.00549.x

ROYER I. \& ZARLOWSKI P. (2001), “Sampling”. In Thietart R.A. \& al. (2001), Doing Management Research, a comprehensive guide (pp. 147-171), Sage. 
SMITH J.A. (2004), "Reflecting on the development of interpretative phenomenological analysis and its contribution to qualitative research in psychology", Qualitative Research in Psychology, 1(1), 39-54.

TASKIN L. \& DIETRICH A. (2016), Management humain (D. Boeck, Ed.), De Boeck Supérieur, Bruxelles.

TAYLOR F.W. (1911), Principes d'organisation scientifique des usines, Dunod et Pinat, Paris.

TENGBLAD S. (2006), "Is there a "new managerial work" ? A comparison with Henry Mintzberg's classic study 30 years later", Journal of Management Studies, 43(7), 1437-1461. http ://doi.org/10.1111/j.1467-6486.2006.00651.x

THEURELLE-STEIN D. \& BARTH I. (2017), « Les soft skills au cœur du portefeuille de compétences des managers de demain », Management \& Avenir, 95(5), 129-151. http://doi.org/10.3917/mav.095.0129.

VAN CAMPENHOUDT L., MARQUET J. \& QUIVY R. (2017), Manuel de recherche en sciences sociales, Dunod.

WEBER M. (1947), The theory of social and economic organizations, New York, Oxford University Press. 